\title{
Acupuncture Treatment in Patient with Uterine Polyp
}

\author{
Prof Dr Jihe Zhu ${ }^{1}$, BSc Blagica Arsovska ${ }^{1,2}$, BSc Kristina Kozovska ${ }^{* 1,3}$ \\ ${ }^{1}$ Faculty of Medical Sciences, University Goce Delchev, Shtip, Republic of Macedonia; \\ ${ }^{2}$ Institute of Biology, Faculty of Natural Sciences and Mathematics, Skopje, Republic of Macedonia \\ ${ }^{3}$ Medicine Faculty, St. Cyril and Methodius University of Skopje, Republic of Macedonia
}

\begin{abstract}
Uterine polyps are benign growths on the inner wall of the uterus, which extend into the uterine cavity. Excessive growth of the mucous membrane of the uterus (endometrium) leads to the formation of polyps on the uterus. Uterine polyps are usually non-cancerous (benign), although some may be carcinogenic or possibly develop over time into cancer. Polyps can be divided into two groups: endo-cervical polyps and endometrial polyps. Uterine polyps most commonly occur in women who undergo menopause or are in postmenopausal period, although polyps may occur in younger women too. In Traditional Chinese Medicine (TCM), the cause for uterine polyps is usually a combination of Blood deficiency, Blood stagnation and Liver/Kidney Deficiency. The aim of the treatment is to move the blood, to make the blood flow free and smooth, resolve the dampness, resolve the blood stagnation, treat the underlying deficiencies and etc. The treated patients is 41 year old woman, diagnosed with $10 \mathrm{~mm}$ uterine polyp. The patient was also diagnosed with infertility and was having irregular periods. The patient was treated with acupuncture in a clinic for TCM and acupuncture in Skopje. In a period of 4 months, 14 treatments were made, indoor, on a room temperature, with duration of the treatments of 30-45 minutes. After the 4th treatment her period was regularized and after 14 treatments the polyp was gone. Acupuncture as a treatment for uterine polyps is safe and effective treatment and gave very satisfying and positive results in our treated patient.
\end{abstract}

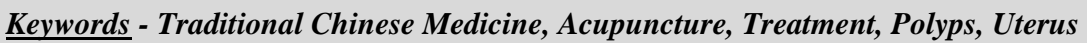

\section{Introduction}

Uterine polyps are benign growths on the inner wall of the uterus, which extend into the uterine cavity. Excessive growth of the mucous membrane of the uterus (endometrium) leads to the formation of polyps on the uterus. Uterine polyps are usually noncancerous (benign), although some may be carcinogenic or possibly develop over time into cancer (pre-cancerous polyps). Uterine polyps can be divided into two groups: endo-cervical polyps and endometrial polyps. Uterine polyps most commonly occur in women who undergo menopause or are in postmenopausal period, although polyps may occur in younger women too.

The polyps are controlled by hormonal factors and are sensitive to estrogen. Polyps can affect fertility by preventing the embryo to implant and embed into the endometrium and make a risk of miscarriage to the growing fetus. To increase the fertility, the polyps can be removed surgically, but more polyps grow back if the underlying cause is left untreated.

In Traditional Chinese Medicine (TCM), the cause for uterine polyps is usually a combination of Blood deficiency, Blood stagnation and Liver or Kidney Deficiency. Symptoms of uterine polyps are: infertility, irregular menstrual periods, menorrhagia, bleeding in between periods, pain and etc. TCM treatments that are used in the uterine polyp treatment are acupuncture and herbs. The herbs and the acupuncture treatment can excellently strengthen the body, move the stagnant Blood and Qi, warm the Kidney Yang, resolve the dampness and improve the Blood and Qi circulation to the uterus. With the treatment can be also stimulated the nerve end to improve the ovarian blood circulation and ovarian function, improve fertility, stop abnormal vaginal bleeding and restore hormonal balance and ovulation. ${ }^{[1]}$

Therefore, the aim of the treatment is to move the Qi and blood, to make the energy flow free and smooth, resolve the dampness, resolve the blood stagnation, treat the underlying deficiencies and etc. $^{[2]}$

\section{Case Report}

The treated patients is 41 year old woman, diagnosed with $10 \mathrm{~mm}$ uterine polyp. The patient was also diagnosed with infertility and was having irregular periods. The patient was treated with acupuncture in a clinic for TCM and acupuncture in Skopje, by a doctor specialist in acupuncture. In a period of 4 months, 14 treatments were made, one treatment per week. Treatments were made indoor, on a room temperature, with duration of the treatments of 30-45 minutes. In the treatment were used fine sterile disposable needles with dimensions $0.25 \times 25 \mathrm{~mm}$ manufactured by Wuijuiang City Medical \& Health Material Co., LTD. Acupuncture points that were treated are: HN1 (SiShenCong), LR1 (Dadun), ST36 (ZuSanLi), LI4 (HeGu), GB34 (YangLingQuan), SP9 (YinLingQuan), SP6 (SanYinJiao), LR3 (TaiChong), RN13 (ZhongWan), RN2 (QuGu), RN7 (QiHai), RN4 (GuanYuan), ST29 (GuiLai), ST25 (TianShu), DU14 (DaZhui), GB20 (FengChi), BL19 (DanShu), BL18 (GanShu), BL21 (WeiShu), BL20 (PiShu), BL25 DaChangShu) and BL32 (CiLiao) 


\section{International Journal of Innovative Research in Medical Science (IJIRMS) Volume 03 Issue 12 Dec 2018, ISSN: 2455-8737, Imp. Factor - 4.102 Available online at - www. ijirms.in}

After the 4th treatment the patient's period was regularized and after 14 treatments the polyp was gone. On figure 1 is shown UV scan of the uterus, done before the treatment on which is visible 10 mm polyp. On figure 2 is shown UV scan after the treatment, where no polyp is visible on the uterus.

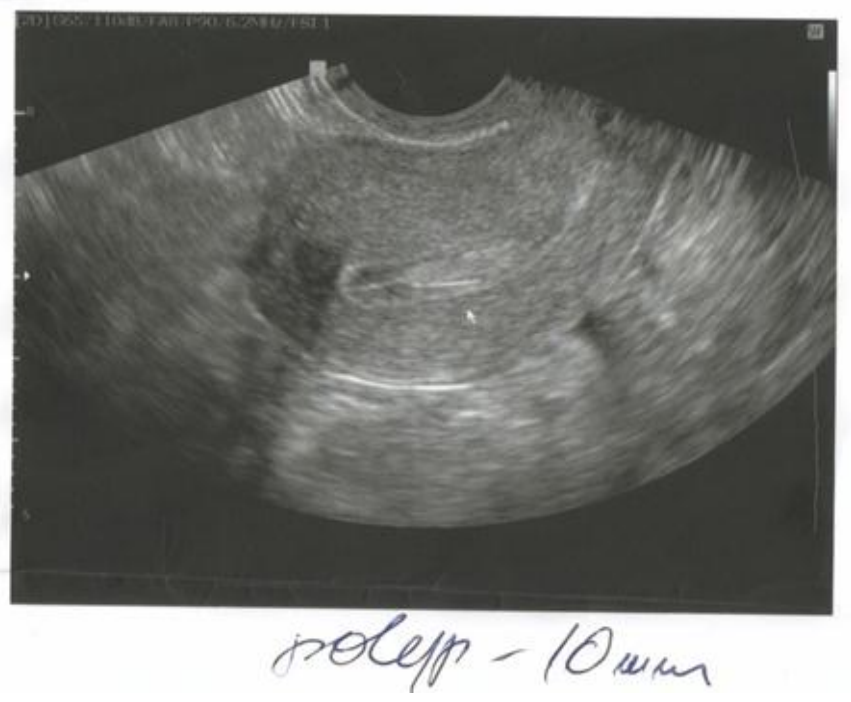

Figure 1: UV scan done before the treatment, showing uterine polyp of $10 \mathrm{~mm}$

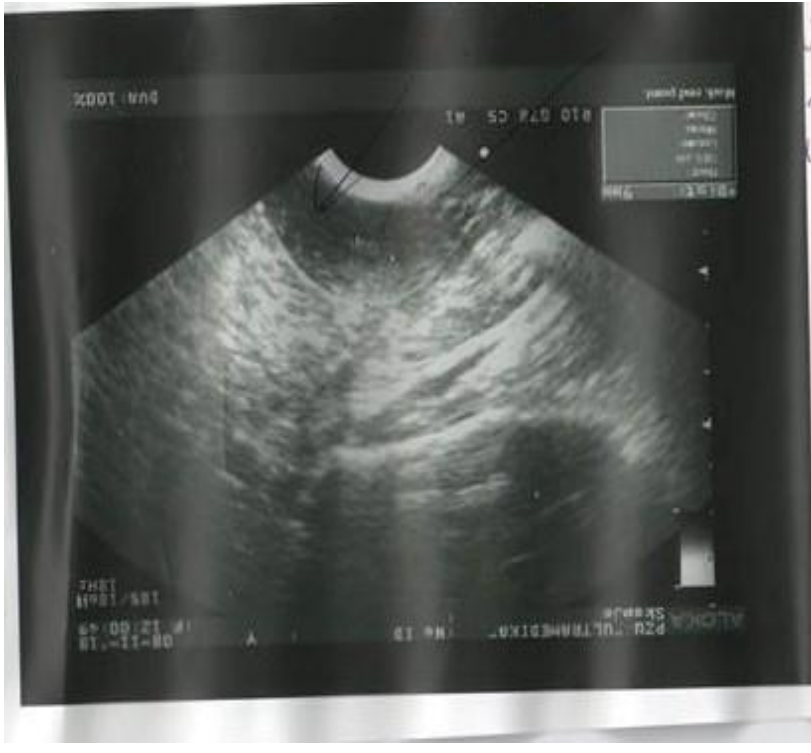

Figure 2: UV scan done after the treatment, with no signs of polyps on the uterus

On table 1 are shown the results from the hormonal test analysis, which are showing normalization of FSH hormone from 19.3 to 9.9 (ref. value 1.98-11.6 mIU/ml).

Table 1: Results from hormonal test done before and after the treatment

\begin{tabular}{|l|c|c|c|c|c|}
\hline & FSH & LH & TSH & Estradiol & Prolactin \\
\hline Before Treatment & 19.3 & 5.6 & 2.85 & 43.6 & 19.4 \\
\hline After Treatment & 9.9 & 6.7 & 2.91 & 129.0 & 23.2 \\
\hline Ref. Value & $1.98-11.6 \mathrm{mIU} / \mathrm{ml}$ & $1.98-11 \mathrm{mIU} / \mathrm{ml}$ & $0.46-4.6 \mathrm{mIU} / \mathrm{ml}$ & $26.5-161 \mathrm{pg} / \mathrm{ml}$ & $3.2-24.3 \mathrm{ng} / \mathrm{ml}$ \\
\hline
\end{tabular}

There are thousands of case reports every day showing the efficacy of TCM in the treatment of many gynecological problems like polyps, PCOS, dysmenorrhea, endometriosis, vaginal discharge and etc. There is a long history of treating gynecological imbalances in women with Chinese herbs and acupuncture, providing an effective and safe treatment. ${ }^{[2,3]}$

Good circulation is vital for the uterine health. In TCM, uterine polyps represent a Blood stagnation pathology. When there is Blood and Qi stagnation, there is poor circulation. ${ }^{[2]}$ Blood stagnation may be caused by stress, injuries, trauma, improper lifestyle, inadequate exercise, poor diet (too much cold food) and other. The symptoms of blood stagnation usually become obvious when the menstrual period comes around. The stagnation in the body causes blockages resulting in hormonal problems. Over time, the blockages can cause changes in the endometrium of the uterus and may cause polyps or fibroids to occur.[4]

Chinese medicine treatment helps in relieving the symptoms, the pain and the discomfort by strengthening and rebalancing the body's energy. The one very important organ related to the women's period is the Liver. When the liver energy is not balanced, it can results in blood stagnation, menstrual blood cloths, uterine cramping, pain, breast distention, moodiness and headache and if by the imbalance are affected the Spleen and Stomach meridians then may occur symptoms like constipation, diarrhea, nausea, abdominal bloating and etc. ${ }^{[5]}$
Acupuncture treatment can help balance the hormones, restore the natural blood flow to the organs, establish a healthy uterus and reproductive system, free up the Qi energy, decrease the polyp growth and many more. ${ }^{[6,7]}$

\section{Conclusion}

Acupuncture as a treatment for uterine polyps is safe and effective treatment and gave very satisfying and positive results in our treated patient.

\section{References}

[1] Ju M; Acupuncture is effective on dysfunctional uterine bleeding and restoring ovulation; 2014 [www.chineseacupuncturelondon.blogspot.com]

[2] Sowter I; Natural Support for Uterine Polyps; 2018 [www.ilanasowter.com]

[3] Zhou J, Qu F; Treating Gynaecological Disorders with Traditional Chinese Medicine: A Review; Afr J Tradit Complement Altern Med. 2009; 6(4): 494-517.

[4] Yu JJ; The Cause of Heavy Periods [Menorrhagia]; 2017 [www.sustainhealth.com.au]

[5] Oudijk C; Treating Painful Periods with Chinese Medicine; 2017 [www.citaoudijk.com]

[6] Craven L; Hormonal health; 2013 [www.novaholisticjournal.com]

[7] Yavin A; Polyps; 2017 [www.acupunctureinny.com] 